\title{
Efficacy and bleeding risk of antithrombin supplementation in septic disseminated intravascular coagulation: a secondary survey
}

\author{
Toshiaki Iba ${ }^{1 *}$, Daizoh Saitoh ${ }^{2}$, Hideo Wada ${ }^{3}$ and Hidesaku Asakura ${ }^{4}$
}

\begin{abstract}
Introduction: In a previous report, we demonstrated a favorable trend for supplementation with antithrombin (AT) concentrate at a dosage of 3,000 IU/day over 1,500 IU/day for the treatment of sepsis-associated disseminated intravascular coagulation (DIC) in patients with an AT activity of $70 \%$ or less. Since the survival difference did not reach statistical significance, we planned to examine the effects in a larger number of cases with severer disease.

Methods: We performed a non-randomized multi-institutional survey. In total, 307 septic DIC patients who had AT activity less than $40 \%$ and who had undergone AT substitution at a dose of either 1,500 IU/day or 3,000 IU/day for three consecutive days were analyzed. Of these, 259 patients received 1,500 IU/day (AT1500 group) and 48 patients received 3,000 IU/day (AT3000 group). The primary efficacy endpoints were recovery from DIC by day 7 and an all-cause mortality on day 28. Adverse bleeding events were also examined. A logistic regression analysis was conducted by using age, sex, body weight, initial AT activity, DIC score, platelet count, coadministration of heparin, recombinant thrombomodulin, suspected source of infection, surgery, and supplemented AT dose.
\end{abstract}

Results: Supplementation significantly decreased the DIC score in the AT3000 group, leading to the superior resolution of DIC, compared with the results in the AT1500 group (66.7\% versus $45.2 \%, P=0.007$ ). In addition, the AT3000 group exhibited a better survival than the AT1500 group ( $77.1 \%$ versus $56.4 \%, P=0.010)$. Bleeding events were observed in 6.96\% (severe bleeding: 3.04\%) in the AT1500 group and 6.52\% (severe bleeding, 4.35\%) in the AT3000 group $(P=1.000$; severe bleeding, $P=0.648)$. A logistic regression analysis revealed that the use of AT3000 (odds ratio (OR), $2.419 ; P=0.025)$, a higher initial platelet count $(\mathrm{OR}, 1.054 ; P=0.027)$, and patient age $(\mathrm{OR}, 0.977 ; P=0.045)$ were significantly correlated with an improved survival.

Conclusions: The AT3000 group exhibited significantly improved rates of survival and recovery from DIC without an increased risk of bleeding, compared with the AT1500 group, among the patients with sepsis-associated DIC and an AT activity of less than $40 \%$.

\section{Introduction}

Severe sepsis is almost consistently associated with a hyper-coagulatory status [1] and frequently leads to disseminated intravascular coagulation (DIC) [2]. Excess clot formation, leading to malcirculation in organs and subsequent organ failure, is driven by activated coagulation, by impaired anticoagulant mechanisms, including the antithrombin (AT) and protein C system, and by the

\footnotetext{
*Correspondence: toshiiba@cf6.so-net.ne.jp

'Department of Emergency and Disaster Medicine, Juntendo University Graduate School of Medicine, 2-1-1 Hongo, Bunkyo-ku, Tokyo 113-8421, Japan

Full list of author information is available at the end of the article
}

depression of the fibrinolytic system [3]. Thus, supplementation with AT concentrates has been considered a rational therapy [4].

Previously, the results of a large-scale randomized controlled trial (RCT) known as KyberSept, which examined the effects and adverse events of high-dose AT for the treatment of severe sepsis, were published [5]. This trial demonstrated that AT did not provide survival benefit and instead increased the risk of bleeding. Since AT, particularly when administered with heparin, increases the risk of bleeding significantly, the international guidelines for severe sepsis recommend "not to use high-dose AT for severe sepsis" [6]. Since the completion of KyberSept, 
no high-powered study has been repeated; however, a systematic review of AT use in patients with DIC with severe sepsis concluded that AT might increase the overall survival [7]. Furthermore, Fourrier [8] demonstrated an improvement in all-cause mortality across subgroups defined according to the DIC status at entry in RCTs of AT and activated protein C. Therefore, it may be too early to conclude that AT is not effective for sepsisassociated DIC.

Recently, the Japanese Association for Acute Medicine (JAAM) DIC study group performed a prospective RCT and reported that $30 \mathrm{IU} / \mathrm{kg}$ per day of AT could decrease the DIC score, compared with a placebo, in septic DIC patients with a baseline AT activity level of between $50 \%$ and $80 \%$, which subsequently resulted in an increase in the DIC resolution rate [9]. Unlike high-dose administration, the supplementation of AT, in which the recovery of AT activity to within the normal range is targeted, has been thought to be beneficial in patients with septic DIC in Japan $[10,11]$. As a result, AT supplementation was included in the Japanese guidelines for sepsis-associated DIC [12]. However, a lack of sufficient evidence has also been pointed out [13]. Under these circumstances, the recent international guideline for the management of DIC has also recommended the use of AT in situations in which the efficacy of such treatment has been proven [13]. Therefore, the examination of the effects and the adverse events associated with this therapy has become an urgent matter.

In a former study, we examined a total of 729 sepsisassociated DIC patients with AT activity levels of $70 \%$ or lower and reported that 3,000 IU/day of AT substitution for 3 days (AT3000) was a significant factor associated with an improvement in survival, compared with 1,500 IU/day of AT substitution (AT1500) (odds ratio (OR) 1.912; $P=0.026)$. However, the survival difference between AT3000 and AT1500 was not statistically significant when examined by using a Fisher's exact test [14]. The other finding in our study was that AT3000 was more effective in a patient population with a lower baseline AT activity. Thus, we further accumulated septic DIC patients with a baseline AT activity of less than $40 \%$. The primary goal of the present study was to confirm an improvement in the resolution of DIC by day 7 and a reduction in all-cause mortality on day 28 .

\section{Materials and methods Definitions}

Both systemic inflammatory response syndrome (SIRS) and sepsis were defined according to the definition of the American College of Chest Physicians/Society of Critical Care Medicine consensus conference [15]. The severity of the illness of the patients was evaluated according to the AT activity [16], JAAM DIC score [17], and Sepsis-Related
Organ Failure Assessment (SOFA) score at the time of enrollment. DIC was defined on the basis of the JAAM DIC criteria [18], and the DIC scores were calculated by using this system. The scoring system consists of the SIRS score, platelet count, fibrin/fibrinogen degradation product (FDP), or D-dimer and prothrombin time ratio; these values were measured in local laboratories. Organ failure was assessed by using the SOFA score [19]. Bleeding events were recorded for 28 days. Bleeding was classified as major if it was intracranial or required a transfusion of at least 3 units of blood. Less-severe bleeding was defined as minor bleeding [5].

\section{Patient selection and data collection}

This survey was performed as a non-randomized, multiinstitutional, post-marketing survey. In total, 307 sepsisassociated DIC patients with an AT activity of less than 40\% who were treated in 217 hospitals between May 2006 and March 2013 were examined in this study. Patients with a history of allergic shock reaction to AT were excluded. The study was conducted in accordance with the Declaration of Helsinki and Good Post-marketing Surveillance Practice (Good Vigilance Practice and Good Postmarketing Study Practice). The institutional review boards that approved this study can be found in the Acknowledgments section. The patients' agreement and consent were obtained when required by the ethics committee of each hospital (between May 2006 and September 2009). All necessary informed consent was obtained from the patients or their relatives by the physician in charge. From September 2009, the agreement was waived since the Japanese Ministry of Health, Labor and Welfare judged that the patients' agreement was not necessary. Patients were registered by the patient registration center, and serial data regarding the AT activity, SIRS score, coagulation markers, and DIC scores were obtained on the day before AT administration (day 0 ) and on days 2, 4, and 7 after AT administration. DIC was judged to have been resolved when the DIC score had decreased to less than 4 as of day 7 . Survival was recorded on day 28. The total bleeding events, including both major and minor bleeding events, were recorded throughout the observation period. Scoring was performed at each hospital.

Either 1,500 IU/day (AT1500) or 3,000 IU/day (AT3000) of AT concentrate (Nihon Pharmaceutical Co. Ltd., Tokyo, Japan) was administered for 3 consecutive days when the patients met the JAAM DIC criteria and had an AT activity level of less than $40 \%$. AT activity was measured at the time of the diagnosis of DIC. The use of AT3000 is approved only for obstetric or surgical DIC patients, and dose adjustment was permitted, depending on the severity. However, since no further details were defined, each doctor decided the dosage on an individual case basis. Standard sepsis care was performed, and platelet concentrate and 
fresh frozen plasma were used as substitution therapy if necessary, as per the guidelines established by the Japanese Ministry of Health, Labor and Welfare.

\section{Laboratory measurements}

Platelet count, coagulation, and fibrinolytic markers were prospectively assessed. When several sets of data were available on a single day, the least favorable measurement value was used for the analysis. The methods that were used to measure platelet count and coagulation included platelet counting (electric impedance method), prothrombin time (scattered light detection), FDP (latex immunoassay), and D-dimer (latex immunoassay). To measure the AT activity, the plasma anti-Factor Xa activity or the antithrombin activity measured in the presence of $0.22 \mathrm{M}$ $\mathrm{NaCl}$, excluding the influence of heparin cofactor II activity, was assessed (chromogenic substrate method, reference intervals: $70 \%$ to $120 \%$ ). All of the measurements were performed by local laboratories.

\section{Statistical analysis}

The paired or unpaired Wilcoxon signed-rank test was applied for two-group comparisons. The proportions were compared by using the chi-square test or the Fisher's exact test. A Kaplan-Meier curve was calculated, and the survival difference was examined by using a log-rank test. The common method of logistic regression analysis (the enter method) used the outcome (survived, 1; died, 0) as the criterion variable and the AT variable dose (3000 IU/ day, 1 ; 1,500 IU/day, 0), patient sex (male, 1; female, 0), age, body weight, baseline AT activity, baseline DIC score, baseline platelet count, respiratory tract infection (yes, 1; no, 0), digestive tract infection (yes, $1 ;$ no, 0 ), urinary tract infection (yes, 1 ; no, 0 ), biliary tract infection (yes, 1 ; no, 0 ), other infection (yes, 1 ; no, 0 ), unknown infection focus (yes, 1 ; no, 0 ), surgery (yes, 1 ; no, 0 ), coadministration of recombinant thrombomodulin (yes, 1 ; no, 0 ), and coadministration of heparins (yes, 1 ; no, 0 ) as explanatory variables. The stepwise method (a forward selection method based on the likelihood ratio) used 28-day survival as the criterion variable and patient sex, age, body weight, baseline AT activity, baseline DIC score, baseline platelet count, respiratory tract infection, digestive tract infection, urinary tract infection, biliary tract infection, other infection, unknown infection focus, surgery and coadministration of recombinant thrombomodulin, coadministration of heparins, and the AT variable dose as explanatory variables. Results were reported as ORs, $P$ values, and 95\% confidence intervals. For all of the reported results, a $P$ value of less than 0.05 was considered to be statistically significant. The above-mentioned analyses were performed by using SPSS 13.0 for MAC OSX (SPSS Inc., Chicago, IL, USA).

\section{Results}

In total, 307 patients were analyzed in the study. Fortyeight patients were treated with AT3000 for 3 days, and 259 patients were treated with AT1500 for 3 days. The mean body weights were $52.3 \mathrm{~kg}$ in the AT1500 group and $52.7 \mathrm{~kg}$ in the AT3000 group $(P=0.863)$.

Table 1 shows the baseline characteristics of the patients. Respiratory tract infection was the most common underlying disease in the AT1500 group, and digestive tract infection was the most frequent in the AT3000 group. In regard to background differences, digestive tract infection was more common in the AT3000 group $(P<0.01)$, and surgery was more common in the AT3000 group $(P<0.01)$. In regard to disease severity, the JAAM DIC score, the AT activity, and the SOFA score were identical between the two groups. The baseline platelet count in the AT1500 group was $6.0 \times 10^{4} / \mathrm{mm}^{3}$ (interquartile range [IQR] 3.15 to 9.1), which was significantly lower than that of the AT3000 group $\left(7.9 \times 10^{4} / \mathrm{mm}^{3}\right.$, IQR 5.0 to 11.4 , $P<0.05)$. Heparin was used in $24.7 \%$ and $16.7 \%$ of the patients in the AT1500 and AT3000 groups, respectively.

Among all of the patients, bleeding events were observed in 19 patients (6.88\%), and 9 (3.26\%) of these were classified as severe. Among the severe bleeding cases, 5 occurred in patients with AT substitution without concomitant unfractionated heparin administration (2.18\%), and 4 occurred in patients with unfractionated heparin administration $(8.51 \%)(P=0.049)$. Among the 9 severe bleeding cases, 7 patients (3.04\%) were treated with AT1500 and 2 were treated with AT3000 (4.35\%); this difference was not significant $(P=0.648)$ (Table 2$)$.

Figure 1 shows the changes in AT activities and the administered AT dose. The AT activities had increased to within the normal range (>80\%) in the AT3000 group, but this level was not reached in the AT1500 group throughout the 7 days of observation. As for DIC resolution, AT3000 resulted in a significant reduction in the DIC score, leading to a better recovery from DIC compared with the AT1500 group (66.7\% versus 45.2\%, $P=$ 0.007 ) (Figure 2). A Kaplan-Meier survival curve was calculated by using data obtained for a maximum of 28 days after the administration of AT. The survival started to decrease from day 3 in the AT1500 group and from day 5 in the AT3000 group, and a significant difference between the groups was recognized $(P=0.007)$ (Figure 3$)$. The AT3000 group exhibited a better survival outcome than the AT1500 group (77.1\% versus 56.4\%, $P=0.010$ [Fisher's exact test], $P=0.007$ [log-rank test]).

The enter method of logistic regression analysis showed that the supplemented AT dose, patient age, and baseline platelet count were significant factors for survival; that is, the AT dose (OR 2.419, $P=0.025$ ), patient age (OR 0.977, $P=0.045$ ), and baseline platelet count (OR 1.054, $P=0.027$ ) contributed to an improvement in survival. In contrast, 
Table 1 Baseline patient demographics

\begin{tabular}{|c|c|c|c|}
\hline & $\begin{array}{l}\text { AT1500 group } \\
n=259\end{array}$ & $\begin{array}{l}\text { AT3000 group } \\
n=48\end{array}$ & $\begin{array}{l}P \\
\text { value }\end{array}$ \\
\hline \multicolumn{4}{|l|}{ Gender } \\
\hline Male & $138(53.3 \%)$ & $27(56.3 \%)$ & \\
\hline Female & $121(46.7 \%)$ & $21(43.8 \%)$ & \\
\hline \multicolumn{4}{|l|}{ Age in years } \\
\hline 14 or under & $1(0.4 \%)$ & $0(0.0 \%)$ & \\
\hline $15-64$ & $60(23.2 \%)$ & $13(27.1 \%)$ & \\
\hline 65 or over & $198(76.4 \%)$ & $35(32.9 \%)$ & \\
\hline \multicolumn{4}{|l|}{$\begin{array}{l}\text { Suspected source } \\
\text { of infection }\end{array}$} \\
\hline Respiratory tract & 85 (32.8\%) & 11 (22.9\%) & \\
\hline Digestive tract & 78 (30.1\%) & 25 (52.1\%) & $<0.05$ \\
\hline Urinary tract & 19 (7.3\%) & $2(4.2 \%)$ & \\
\hline Biliary tract & $28(10.8 \%)$ & $4(8.3 \%)$ & \\
\hline Others & $26(10.0 \%)$ & $6(12.5 \%)$ & \\
\hline Unknown & $35(13.5 \%)$ & $2(4.2 \%)$ & \\
\hline Surgery & $84(34.0 \%)$ & $27(56.3 \%)$ & $<0.05$ \\
\hline \multicolumn{4}{|l|}{ Baseline AT activity } \\
\hline$<30 \%$ & $88(34.0 \%)$ & 19 (39.6\%) & \\
\hline $30 \%$ & $171(66.0 \%)$ & $29(60.4 \%)$ & \\
\hline SD & $31.02 \pm 7.25$ & $29.57 \pm 8.22$ & \\
\hline \multicolumn{4}{|l|}{ Baseline DIC score } \\
\hline 4.5 & $143(55.2 \%)$ & $26(54.2 \%)$ & \\
\hline 6 & $48(18.5 \%)$ & $10(20.8 \%)$ & \\
\hline 7.8 & $68(26.3 \%)$ & $12(25.0 \%)$ & \\
\hline SD & $5.6 \pm 1.3$ & $5.7 \pm 1.4$ & \\
\hline \multicolumn{4}{|l|}{ Baseline SOFA score } \\
\hline $0-6$ & $27(26.5 \%)$ & $5(31.3 \%)$ & \\
\hline $7-12$ & $46(45.1 \%)$ & $9(56.3 \%)$ & \\
\hline $13-24$ & $29(28.4 \%)$ & $2(12.5 \%)$ & \\
\hline SD & $10.1 \pm 4.7$ & $7.7 \pm 4.3$ & \\
\hline $\begin{array}{l}\text { Median baseline platelet } \\
\text { count, } \times 10^{4} / \mathrm{mm}^{3}\end{array}$ & $6.0($ IQR 3.15-9.1) & 7.9 (IQR 5.0-11.4) & $<0.05$ \\
\hline $\begin{array}{l}\text { Median baseline FDP, } \\
\mu \mathrm{g} / \mathrm{mL}\end{array}$ & $\begin{array}{l}\text { 22.1 (IQR 11.5- } \\
37.8)\end{array}$ & $\begin{array}{l}23.7 \text { (IQR 5.0- } \\
11.4)\end{array}$ & $<0.05$ \\
\hline Heparin & $64(24.7 \%)$ & $8(16.7 \%)$ & \\
\hline Thrombomodulin & 15 (5.8\%) & $3(6.3 \%)$ & \\
\hline
\end{tabular}

Values are presented as number (percentage) unless otherwise indicated. AT, antithrombin; DIC, disseminated intravascular coagulation; FDP, fibrin/fibrinogen degradation product; IQR, interquartile range; SD, standard deviation; SOFA, Sepsis-Related Organ Failure Assessment.

neither digestive tract infection nor surgery contributed to the survival (Table 3). A forward selection method based on the likelihood ratio of logistic regression analysis revealed that supplemented AT dose, baseline platelet count and the coadministratrion of recombinant thrombomodulin were significant factors for survival; that is,
Table 2 Bleeding events

\section{Total}

Any bleeding Major bleeding

\begin{tabular}{lcccc}
\hline $\begin{array}{l}\text { Without concomitant unfractionated } \\
\text { heparin }(n=229)\end{array}$ & 13 & $(5.68 \%)$ & 5 & $(5.68 \%)$ \\
$\begin{array}{l}\text { With concomitant unfractionated } \\
\text { heparin }(n=47)\end{array}$ & 6 & $(12.77 \%)$ & 4 & $(8.51 \%)$ \\
Total $(n=276)$ & 19 & $(6.88 \%)$ & 9 & $(3.26 \%)$ \\
\hline
\end{tabular}

\section{AT1500 group}

Any bleeding Major bleeding

\begin{tabular}{lllll}
\hline $\begin{array}{l}\text { Without concomitant unfractionated } \\
\text { heparin }(n=170)\end{array}$ & 6 & $(3.53 \%)$ & 3 & $(1.76 \%)$ \\
$\begin{array}{l}\text { With concomitant unfractionated } \\
\text { heparin }(n=60)\end{array}$ & 10 & $(16.67 \%)$ & 4 & $(6.67 \%)$ \\
Total $(n=230)$ & 16 & $(6.96 \%)$ & 7 & $(3.04 \%)$ \\
\hline
\end{tabular}

\section{AT3000 group}

Any bleeding Major bleeding

Without concomitant unfractionated $3 \quad$ (7.89\%) $2 \quad$ (5.26\%) heparin $(n=38)$

$\begin{array}{lllll}\text { With concomitant unfractionated } & 0 & (0.0 \%) & 0 & (0.0 \%)\end{array}$ heparin $(n=8)$

$\begin{array}{lllll}\text { Total }(n=46) & 3 & (6.52 \%) & 2 & (4.35 \%)\end{array}$

Values are presented as number (percentage).

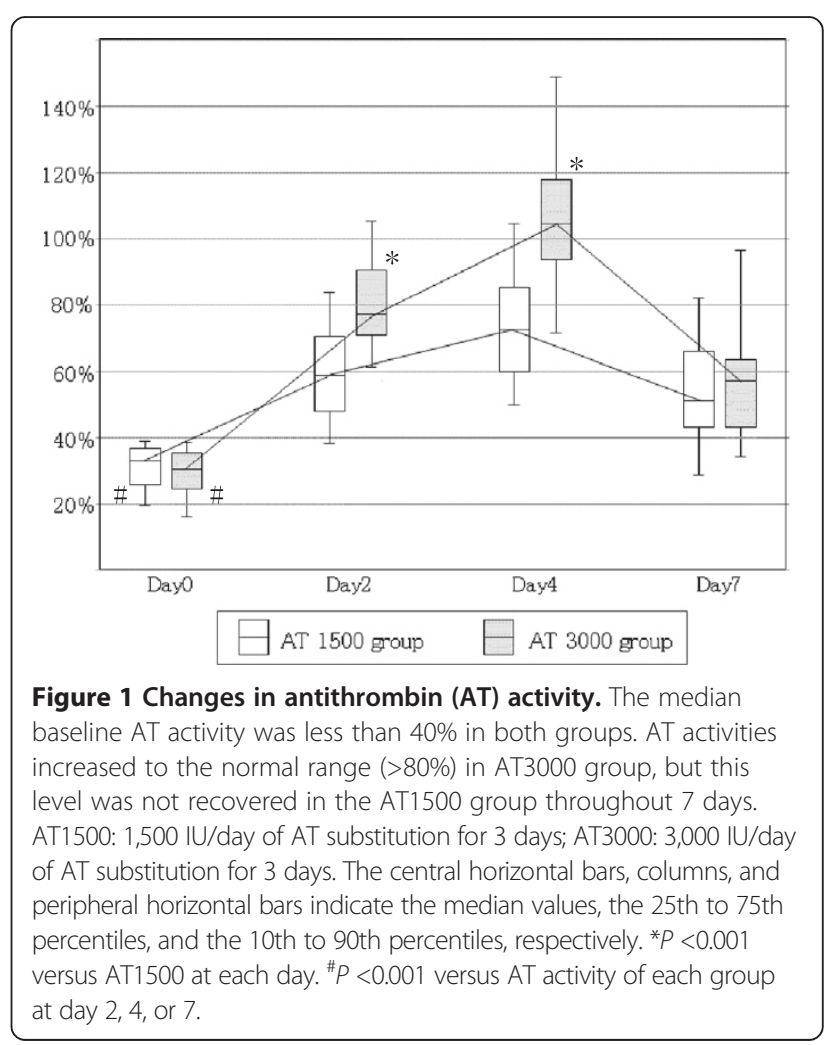




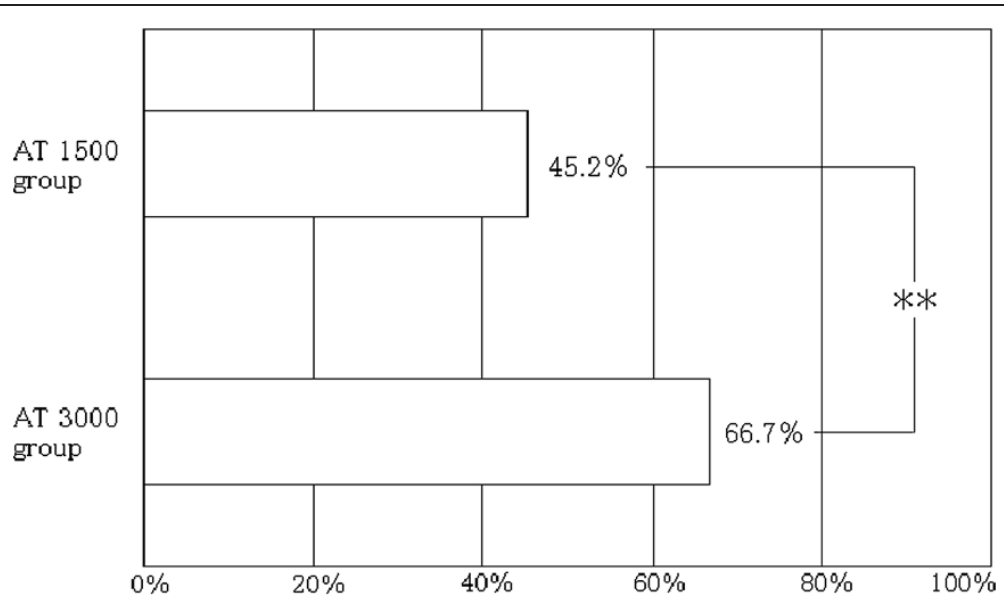

Figure 2 Comparison of disseminated intravascular coagulation (DIC) resolution rates. The DIC resolution rates on day 7 were $45.2 \%$ in the group with 1,500 IU/day of antithrombin substitution for 3 days (AT1500 group) and 66.7\% in the group with 3,000 IU/day of antithrombin substitution for 3 days (AT3000 group). The difference in the DIC resolution rates between these two groups was significant $\left({ }^{* *} P=0.007\right.$, Fisher's exact test).

the AT dose (OR 2.387, $P=0.020)$, baseline platelet count (OR 1.071, $P=0.002$ ), and recombinant thrombomodulin (OR 3.853, $P=0.038$ ) contributed to improved survival (Table 4).

\section{Discussion}

The effectiveness of a supplementation dose of AT for septic DIC is controversial. Sawamura and colleagues [20] performed a retrospective analysis in 23 DIC patients and reported that $60 \mathrm{IU} / \mathrm{kg}$ per day of AT did not show any advantages for the recovery of platelet counts, coagulation and fibrinolytic markers, and DIC scores, compared with $30 \mathrm{IU} / \mathrm{kg}$ per day of AT. In the current survey, AT3000 was found to improve the outcome of sepsis-associated DIC patients significantly, compared with AT1500. The differences between their study and ours were not only the numbers of patients but also the initial AT activity. Since the first survey demonstrated that the efficacy of AT3000 was more prominent in lower AT activity, we focused on the patients with AT activities of less than $40 \%$ in the present study.

As for the relationship between the recovered level of AT activity and efficacy, Gando and colleagues [21] retrospectively evaluated the changes in the AT activity after

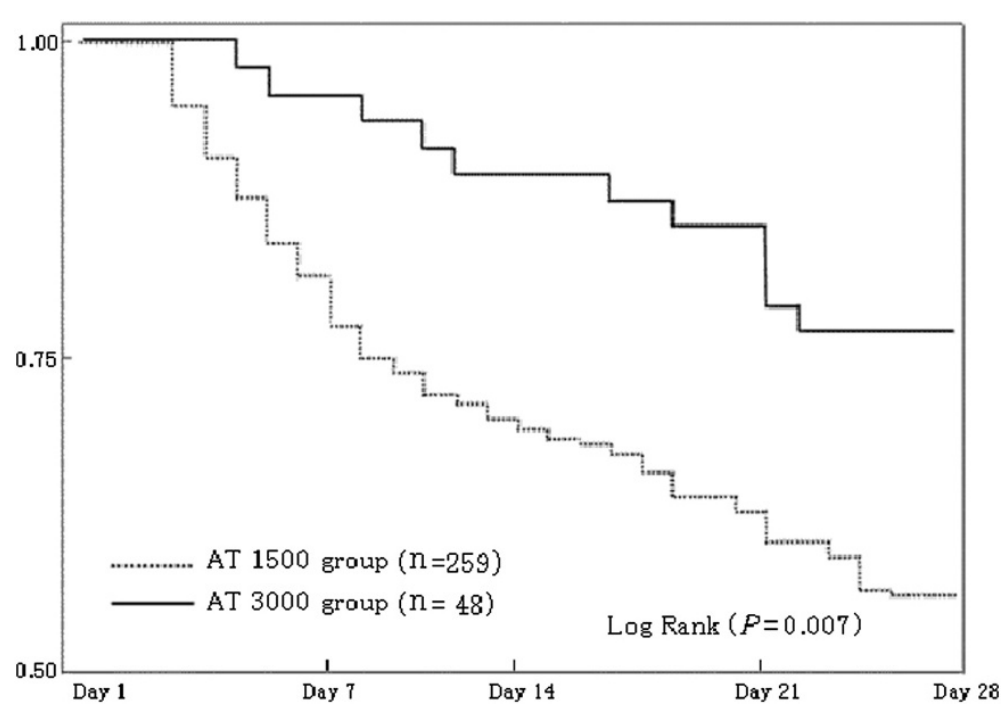

Figure 3 Comparison of survival. A Kaplan-Meier curve was calculated to compare the survival between the patients treated with 1,500 IU/day of antithrombin and the patients treated with 3,000 IU/day of antithrombin. The survival started to decrease from day 3 in the AT1500 group and from day 5 in the in the AT3000 group. A significant difference between the groups was recognized on day 28 ( $P=0.007$, log-rank test). 
Table 3 Relationship between outcome (28-day survival) and various factors using the enter method of logistic regression analysis

\begin{tabular}{|c|c|c|c|}
\hline & $\begin{array}{l}\text { Odds } \\
\text { ratio }\end{array}$ & $P$ value & $\begin{array}{l}95 \% \text { confidence } \\
\text { interval }\end{array}$ \\
\hline \multicolumn{4}{|l|}{ AT dose: } \\
\hline 3,000 versus $1,500 \mathrm{IU}$ & 2.414 & 0.025 & $1.115-5.226$ \\
\hline Gender & 0.746 & 0.296 & $0.432-1.291$ \\
\hline Age & 0.977 & 0.045 & $0.955-0.999$ \\
\hline Body weight & 0.998 & 0.901 & $0.974-1.023$ \\
\hline Baseline AT activity & 1.004 & 0.822 & $0.969-1.041$ \\
\hline Baseline DIC score & 0.875 & 0.198 & $0.714-1.072$ \\
\hline Heparin & 0.808 & 0.499 & $0.435-1.500$ \\
\hline Recombinant thrombomodulin & 3.639 & 0.057 & $0.960-13.790$ \\
\hline Baseline platelet count & 1.054 & 0.027 & $1.006-1.104$ \\
\hline Respiratory tract infection & 0.632 & 0.492 & $0.170-2.343$ \\
\hline Digestive tract infection & 1.034 & 0.960 & $0.279-3.832$ \\
\hline Urinary tract infection & 3.019 & 0.140 & $0.695-13.115$ \\
\hline Biliary tract infection & 1.208 & 0.791 & $0.300-4.871$ \\
\hline Other infection & 0.799 & 0.772 & $0.175-3.640$ \\
\hline Unknown infection focus & 0.901 & 0.891 & $0.203-3.995$ \\
\hline Surgery & 0.929 & 0.818 & $0.498-1.733$ \\
\hline
\end{tabular}

AT, antithrombin; DIC, disseminated intravascular coagulation.

supplementation with a fixed dose of AT (1,500 IU/day or $30 \mathrm{IU} / \mathrm{kg}$ per day) and reported that patients who achieved an AT activity level of more than $60 \%$ had a better outcome. In our survey, the AT activity reached over $80 \%$ in the AT3000 group but was around $60 \%$ in the AT 1500 group. However, whether the recovered AT activity was a result of the supplementation or the cause of the improvement in survival is not clear. A target activity-oriented AT supplementation study should be performed to address this issue.

The main concern regarding the use of AT is the possible increase in the risk of bleeding. When high-dose AT, in particular, was administered with either unfractionated or low-molecular-weight heparin, the number of bleeding events increased significantly $(23.8 \%$ for AT with heparin

Table 4 Relationship between the outcome (28-day survival) and various factors using the forward selection method based on the likelihood ratio of logistic regression analysis

\begin{tabular}{llll}
\hline & $\begin{array}{l}\text { Odds } \\
\text { ratio }\end{array}$ & $\boldsymbol{P}$ value & $\begin{array}{l}\mathbf{9 5 \%} \text { confidence } \\
\text { interval }\end{array}$ \\
\hline Baseline platelet count & 1.071 & 0.002 & $1.025-1.119$ \\
Recombinant thrombomodulin & 3.853 & 0.039 & $1.072-13.855$ \\
Antithrombin dose & & & \\
3,000 versus 1,500 IU & 2.387 & 0.020 & $1.144-4.983$ \\
\hline
\end{tabular}

versus 13.5 for placebo; $P<0.001$ ) in KyberSept [5]. In contrast, heparin use did not affect the incidence of bleeding events in our former survey. A total of 193 out of 729 patients $(26.5 \%)$ received heparin, and neither the incidences of bleeding events $(6.55 \%$ with heparin versus $6.51 \%$ without heparin) nor the increase of serious bleeding events (1.79\% with heparin versus $1.68 \%$ without heparin) differed between patients treated with and those treated without heparin. In the present study, the number of bleeding events was more than two times higher in patients with concomitant heparin use, compared with those receiving AT alone. We suspect that the severity of sepsis and the degree of coagulation disorder might have influenced this difference, but further examination is needed to clarify this issue. In regard to the bleeding risk without heparin treatment, the rate was not significantly higher than that observed in the former survey regardless of the increase in coagulation disorder. There was no intracranial hemorrhage and deadly bleeding in this study; however, it should be kept in mind that the rate of severe bleeding was doubled in the present study.

Interestingly, the coadministration of recombinant thrombomodulin was revealed to be another significant factor for survival by the forward selection method of logistic regression analysis. Because both AT and thrombomodulin activities are known to decrease significantly during severe sepsis and their mechanisms of action are independent, a combination therapy could potentially be beneficial. On the basis of this hypothesis, we examined the combination effects in the lipopolysaccharide-challenged animal model and reported that the combination therapy attenuates organ damage, causes histologic changes, and leads to an improvement in survival $[22,23]$. However, the efficacy has not been proven in the clinical field yet. Furthermore, since the $P$ value did not reach significant in the enter method, this issue should be discussed in the next study.

Finally, the present study had several limitations. First, the superiority of the AT3000 regimen might be related to the surgery rather than the AT volume. As was shown by the stepwise logistic regression analysis, surgery was a significant factor in the use of AT3000, and this result can be explained by the Japanese health-care system. Since DIC patients who require surgical treatment and obstetric DIC patients have lower AT activity levels, the use of AT3000 is approved for these patients. To answer the question of whether the surgery itself, rather than the AT3000 treatment, might have improved the outcome, it should be noted that since the enter method of logistic regression analysis revealed that surgery was not a significant explanatory variable for the outcome, the AT3000 regimen might be responsible for the improvement in survival. A second limitation is that the dose allocations were not randomly performed. Although the baseline SOFA score was 
not different between the groups, the ratio of very severe cases (SOFA score $>12$ ) was higher in the AT1500 group than in the AT3000 group (28.4\% versus $12.5 \%$ ), and this might have affected the results. Third, a placebo control was not included in the study. Fourth, the patient number in the AT3000 group was relatively small. In addition, we cannot eliminate the influence of the higher baseline platelet count in the AT3000 group. Therefore, we think that the survey should be continued.

\section{Conclusions}

The supplementation of AT at a dose of 3,000 IU/day (compared with 1,500 IU/day) for 3 days resulted in the effective modulation of sepsis-associated DIC resolution and an improvement in the 28-day survival outcome without increasing the risk of bleeding in DIC patients with a baseline AT activity level of less than $40 \%$. Although the results of the present study may provide a rationale for the use of a supplemental dose of AT, a large-scale RCT addressing the efficacy and bleeding risk of AT for DIC in patients with severe sepsis is required.

\section{Key messages}

- AT supplementation at 3,000 IU/day improved the outcome of sepsis-associated DIC in patients with a baseline AT activity level of less than $40 \%$, compared with 1,500 IU/day of AT.

- AT supplementation at 3,000 IU/day does not increase the bleeding risk, compared with AT supplementation at 1,500 IU/day.

- The concomitant use of heparin may increase the bleeding risk.

\section{Abbreviations}

AT: antithrombin; DIC: disseminated intravascular coagulation; FDP: fibrin/ fibrinogen degradation product; IQR: interquartile range; JAAM: Japanese Association for Acute Medicine; OR: odds ratio; RCT: randomized controlled trial; SIRS: systemic inflammatory response syndrome; SOFA: Sepsis-Related Organ Failure Assessment.

\section{Competing interests}

$\mathrm{TI}, \mathrm{DS}, \mathrm{HA}$, and HW are the members of the Japanese Society of Thrombosis Hemostasis/DIC subcommittee. This work was performed as post-marketing surveillance by Nihon Pharmaceutical Co. Ltd., which operated the patient registration center and collected the data from each institute. The authors declare that they have no competing interests.

\section{Authors' contributions}

$\mathrm{TI}, \mathrm{DS}, \mathrm{HA}$, and HW contributed to the conceptual design of the survey. DS performed the statistical analysis. TI and DS wrote the manuscript. All authors read and approved the final manuscript.

\section{Acknowledgments}

The institutional review boards of the following hospitals approved the present survey: University of Tsukuba Hospital, Showa University Hospital, Showa University Toyosu Hospital, Toho University Omori Medical Center, Yokosuka Kyosai Hospital, Aichi Medical University, Mie University Hospital, Tosei General Hospital, Kyoto Prefectural University of Medicine University Hospital, Kanazawa University Hospital, Tokushima University Hospital, Kochi Medical School Hospital, National Hospital Organization Beppu Medical
Center, Asahikawa Medical University Hospital, Jichi Medical University Hospital, Gunma University Hospital, Niigata Tokamachi Hospital, Mie University Hospital, and Hiroshima University Hospital. The ethics committees of the following hospitals permitted the survey without discussion: Tokyo Medical University Hachioji Medical Center, Nippon Medical School Tama Nagayama Hospital, the Hospital of Hyogo College of Medicine, Osaka Mishima Emergency Critical Care Center, Sugita Genpaku Memorial Obama Municipal Hospital, Nishio Municipal Hospital, Okazaki City Hospital, Tokyo Women's Medical University Yachiyo Medical Center, Yonezawa City Hospital, and the National Hospital Organization Asahikawa Medical Center. The authors thank all of the institutes that cooperated with this study and thank Makoto Arakawa and Kenji Kinoshita for collecting the data.

\section{Author details}

'Department of Emergency and Disaster Medicine, Juntendo University Graduate School of Medicine, 2-1-1 Hongo, Bunkyo-ku, Tokyo 113-8421, Japan. ${ }^{2}$ Division of Traumatology, Research Institute, National Defense Medical College, 3-2 Namiki, Tokorozawa-shi, Saitama 359-8513, Japan. ${ }^{3}$ Department of Molecular and Laboratory Medicine, Mie University Graduate School of Medicine, Tsu, Mie 514-8507, Japan. ${ }^{4}$ Department of Internal Medicine III, Kanazawa University School of Medicine, 13-1 Takaramachi, Kanazawa, Ishikawa 920-8641, Japan.

Received: 22 December 2013 Accepted: 14 August 2014

Published online: 15 September 2014

\section{References}

1. Kinasewitz GT, Yan SB, Basson B, Comp P, Russell JA, Cariou A, Um SL, Utterback B, Laterre PF, Dhainaut JF: Universal changes in biomarkers of coagulation and inflammation occur in patients with severe sepsis, regardless of causative micro-organism. Crit Care 2004, 8:R82-R90.

2. Levi M, van der Poll T: Inflammation and coagulation. Crit Care Med 2010, 38:S26-S34.

3. Angus DC, van der Poll T: Severe sepsis and septic shock. N Engl J Med 2013, 369:840-851.

4. Levi M: Pathogenesis and treatment of disseminated intravascular coagulation in the septic patient. J Crit Care 2001, 16:167-177.

5. Warren BL, Eid A, Singer P, Pillay SS, Carl P, Novak I, Chalupa P, Atherstone A, Pénzes I, Kübler A, Knaub S, Keinecke HO, Heinrichs $H$, Schindel F, Juers M, Bone RC, Opal SM: Caring for the critically ill patient: High-dose antithrombin III in severe sepsis: a randomized controlled trial. JAMA 2001, 286:1869-1878.

6. Dellinger RP, Levy MM, Rhodes A, Annane D, Gerlach H, Opal SM, Sevransky JE, Sprung CL, Douglas IS, Jaeschke R, Osborn TM, Nunnally ME, Townsend SR, Reinhart K, Kleinpell RM, Angus DC, Deutschman CS, Machado FR, Rubenfeld GD, Webb SA, Beale RJ, Vincent JL, Moreno R: Surviving sepsis campaign: international guidelines for management of severe sepsis and septic shock: 2012. Crit Care Med 2013, 41:580-637.

7. Wiedermann CJ, Kaneider NC: A systematic review of antithrombin concentrate use in patients with disseminated intravascular coagulation of severe sepsis. Blood Coagl Fibrinolysis 2006, 17:521-526.

8. Fourrier F: Severe sepsis, coagulation, and fibrinolysis: dead end or one way? Crit Care Med 2012, 40:2704-2708.

9. Gando S, Saitoh D, Ishikura H, Ueyama M, Otomo Y, Oda S, Kushimoto S, Tanjoh K, Mayumi T, Ikeda T, Iba T, Eguchi Y, Okamoto K, Ogura H, Koseki K, Sakamoto Y, Takayama Y, Shirai K, Takasu O, Inoue Y, Mashiko K, Tsubota T, Endo S: A randomized, controlled, multicenter trial of the effects of antithrombin on disseminated intravascular coagulation in patients with sepsis. Crit Care 2013, 17:R297.

10. Iba T, Nagaoka I, Boulat M: The anticoagulant therapy for sepsis-associated disseminated intravascular coagulation. Thromb Res 2013, 131:383-389.

11. Iba T: Harmonized guidance for DIC from the ISTH and the current status of anticoagulant therapy in Japan. J Thromb Haemost 2013, 11:2076-2078.

12. Wada H, Asakura H, Okamoto K, Iba T, Uchiyama T, Kawasugi K, Koga S, Mayumi T, Koike K, Gando S, Kushimoto S, Seki Y, Madoiwa S, Maruyama I, Yoshioka A: Expert consensus for the treatment of disseminated intravascular coagulation in Japan. Thromb Res 2010, 125:6-11.

13. Wada H, Thachil J, Di Nisio M, Mathew P, Kurosawa S, Gando S, Kim HK, Nielsen JD, Dempfle CE, Levi M, Toh CH: The Scientific Standardization Committee on DIC of the International Society on Thrombosis Haemostasis. Guidance for diagnosis and treatment of DIC from 
harmonization of the recommendations from three guidelines. $J$ Thromb Haemost 2013, 11:761-767.

14. Iba T, Saito S, Wada H, Asakura H: Efficacy and bleeding risk of antithrombin supplementation in septic disseminated intravascular coagulation: A prospective multicenter survey. Thromb Res 2012, 130:e129-e133.

15. American College of Chest Physicians/Society of Critical Care Medicine Consensus Conference: definitions for sepsis and organ failure and guidelines for the use of innovative therapies in sepsis. Crit Care Med 1992, 20:864-874.

16. Iba T, Gando S, Murata A, Kushimoto S, Saitoh D, Eguchi Y, Ohtomo Y, Okamoto K, Koseki K, Mayumi T, Ikeda T, Ishhikura H, Ueyama M, Ogura Y, Endo S, Shimazaki S: Predicting the severity of SIRS-associated coagulopathy with hemostatic molecular markers and vascular endothelial injury markers. J Trauma 2007, 63:1093-1098.

17. Gando S, Saitoh D, Ogura H, Mayumi T, Koseki K, Ikeda T, Ishikura H, Iba T, Ueyama M, Eguchi Y, Ohtomo Y, Okamoto K, Kushimoto S, Endo S, Shimazaki S: Natural history of disseminated intravascular coagulation diagnosed based on the newly established diagnostic criteria for critically ill patients: results of a multicenter, prospective survey. Crit Care Med 2008, 36:145-150.

18. Gando S, Iba T, Eguchi Y, Ohtomo Y, Okamoto K, Koseki K, Mayumi T, Murata A, Ikeda T, Ishikura H, Ueyama M, Ogura H, Kushimoto S, Saitoh D, Endo S, Shimazaki S: A multicenter, prospective validation of disseminated intravascular coagulation diagnostic criteria for critically ill patients: comparing current criteria. Crit Care Med 2006, 34:625-631.

19. Vincent JL, de Mendonça A, Cantraine F, Moreno R, Takala J, Suter PM, Sprung $\mathrm{CL}$, Colardyn F, Blecher S: Use of the SOFA score to assess the incidence of organ dysfunction/failure in intensive care units: results of a multicenter, prospective study. Crit Care Med 1998, 26:1793-1800.

20. Sawamura A, Gando S, Hayakawa M, Hoshino H, Kubota N, Sugano M: Effects of antithrombin III in patients with disseminated intravascular coagulation diagnosed by newly developed diagnostic criteria for critical illness. Clin Appl Thromb Hemost 2009, 15:561-566.

21. Gando S, Sawamura A, Hayakawa M, Hoshino H, Kubota N, Oshiro A: First day dynamic changes in antithrombin III activity after supplementation have a predictive value in critically ill patients. Am J Hematol 2006, 81:907-914

22. Iba T, Nakarai E, Takayama T, Nakajima K, Sasaoka T, Ohno Y: Combination effect of antithrombin and recombinant human soluble thrombomodulin in an LPS induced rat sepsis model. Crit Care 2009, 13:R203-R209.

23. Iba T, Miki T, Hashiguchi N, Yamada A, Nagaoka I: Combination of antithrombin and recombinant thrombomodulin attenuates leukocyte-endothelial interaction and suppresses the increase of intrinsic DAMPs in endotoxemic rats. J Surg Res 2014, 187:581-586.

\section{Submit your next manuscript to BioMed Central and take full advantage of:}

- Convenient online submission

- Thorough peer review

- No space constraints or color figure charges

- Immediate publication on acceptance

- Inclusion in PubMed, CAS, Scopus and Google Scholar

- Research which is freely available for redistribution 